\title{
Why and in what sense do conference interpreters need special software?
}

\author{
Anja Rütten \\ Fachhochschule Köln
}

Information society, digitalisation, software solutions for everybody. Also for conference interpreters? What are the special characteristics of an interpreter's workflow that require specialised software? Does this specialised software already exist? After having a look at scientific findings and existing solutions in this field, this article shows the differences between the software requirements of interpreters and those of translators or terminologists. On the basis of these considerations, a model for a special software solution for conference interpreters is presented.

\section{Introduction}

Interpreters - just like many other professionals - have to deal with an abundance of information from many different sources, and they do so under extreme circumstances: When preparing for the subject of a given conference (usually highly specialised, with subjects varying widely from one conference to the next), they need this very special and reliable information in the respective languages and well before the conference starts. Interpreters therefore need a system that serves their professional purposes by accessing, categorising and representing the required content and linguistic information quickly and precisely according to individually pre-set criteria. This helps them memorise relevant information under time pressure, permits intuitive information research while interpreting (in the booth) and supports efficient follow-up and updating of the personal information database afterwards. This paper will suggest a model that could satisfy the special needs of interpreters, being complex enough to satisfy their specific and individual requirements as well as simple and user-friendly enough to produce the necessary output easily and quickly, as it will only be useful if it does not cause additional complications.

\section{State of the art in research}

As to the methods and tools for information management before, during and after a conference, there are a number of articles as well as some dissertations and $\mathrm{PhD}$ theses written by conference interpreters. However, many of these texts are descriptions of personal experience or statements that do not necessarily have a systematic scientific foundation. Although in many cases these authors are experienced simultaneous interpreters and their affirma- 
tions are of undoubted relevance for the interpreting community, they cannot always be relied upon as scientific evidence in order to draw up a model solution for interpreters' knowledge management systems.

In my dissertation (Rütten 2000) I started to investigate the basis for optimum information management before, during and after a conference with the main focus on conference preparation as a means to facilitate information handling during interpretation - and then drew up a list of requirements and a basic structure for an appropriate software model. I looked into interpre-tation research findings as well as neighbouring disciplines like termino-logy, foreign language teaching and the psychology of learning in order to find out about specific needs. As I had expected, much evidence could be found about characteristics and problems of conference preparation for interpretation.

Interpreting is a very knowledge-intensive activity. Preparing for it means self-organised learning. It is important to systematise this process of information retrieval from huge quantities of data (material received from the customer or colleagues, the interpreter's own existing data from, for example, previous conferences and other sources like the internet), which is repeated over and over again with constantly changing, very specific subjects and under great time pressure. The following points are of special relevance.

It is crucial to prepare on the semantic level to make sure the meaning of what the speaker is saying is understood. This is something that should not be taken for granted considering the fact that today conferences may be on very diverse subjects ranging from sugar beet harvesting to anti-aircraft missiles and discussions about accounting problems in an international company. In this context, efficient background document research, classification and storage (in order to be able to find the material back when working more than once for the same customer) are essential. On the other hand, when it comes to extracting or finding and managing terminology, instead of recording mere vocabulary, it is also important to include additional information like semantic and conceptual aspects and relations (definitions, hyponyms, hyperonyms, antonyms, meronyms, holonyms etc.).

When working through technical texts it will be easier to concentrate on the meaning of the text if relevant terminology has already been extracted (semi-)automatically. Copying terms from the text onto a list is quite time-consuming. Automatically generated term lists will always have to be revised by the user, but even so, the "rough" work has already been done and the interpreter, when reading the list, can concentrate on those terms that are relevant and important to remember or which he/she already knows.

Working with multilingual word lists is widely disapproved of among translators or terminologists. These simple lists lack any kind of additional information concerning grammar, meaning, reliability etc. They often even seem inconsistent and unstructured or do not correspond to the official, standardised terms. However, several interpreters have already underlined their usefulness, and there are occasions where such lists may very well serve the 
special purpose of conference interpreters, which simply entails finding the right word at the right moment. Unfortunately, they cannot be relied upon to always deliver the needed information immediately ("Crankshaft flywheel? - Wait a second, I think I have this one on a list from last month's conference."). It should be noted that interpreters' requirements are quite different from those of translators, terminologists or lexicographers and that they need something much less complete, but that, on the other hand, something more complete than what is found currently in practice might still be useful, e.g. including all entries in one database or at least under one interface.

The existence of equivalences between languages is normally denied; however, simultaneous interpretation makes it necessary to automate parts of the transfer, so that in a limited number of cases (names, some technical terms etc.) elements of different languages can be considered equivalent at least for the context of a special conference.

Interpreters will never be machines spitting out word equities; however, in order to retain very technical terminology within (and for) a short period of time, some automation of word pairs may be necessary, for example in the case of complicated new expressions, so that when talking about things like Ansaugkrümmerunterdruckumformer or hydroxyanthraquinone, the interpreter still gives a convincing and professional rendering. A distinction can be made between transfers that can or should be automated between two or more languages - "1 to 1 " (or near) correspondences - and transfers that must be done consciously, which take up a considerable part of the attention available. An ideal interpreters' program should be designed accordingly.

Memorising terms and other language elements could be more systematic and efficient when controlled by means of a program. It could also be helpful not only to train terms visually, but also acoustically - which, apart from addressing a different perception channel and enhancing integration into the memory structure, also corresponds to the real working conditions.

There are basically two levels of preparation: long-term preparation - i.e. the cultivation of the working languages (active and passive, mother tongue and foreign languages), especially in terms of general language - and short-term, technical conference preparation (special language).

Findings in learning psychology suggest that the structuring and classification of information in different categories enhances retrieval. Especially in the situation of simultaneous interpretation less attention can be dedicated to the task of word retrieval, so it must work smoothly. The information inventory of a database should be structured individually, according to the particular interpreter's working and memory structures into which the new information must be integrated, in order to make it retrievable not only from the computer but from the individual's memory. A well-structured information stock on your hard disk will also aid a well-structured memory. When memorising linguistic and technical knowledge, classifica- 
tion plays an essential role; it can be helpful to work in different categories, but it may also be good to deliberately memorise elements in a disordered way.

There is a huge amount of potentially useful additional information and characteristics for the entries in a terminology database. Thus, space for many additional items should theoretically be provided in each entry, even if not every interpreter makes use of the whole range of possible additional items, and additional information is not entered for each and every entry. A link between a special term and a corresponding picture can provide illustration, which aids memorisation. Notes like "difficult", "very important" or "useful/nice formulation" (to improve style) as well as degrees of reliability or information sources are examples of useful items of information. But I would say that filing more than what is deemed beneficial by the user would be counter-productive and would result in interpreters not using this (or any other) system.

The above summarises the characteristics and difficulties of interpreters' information management identified in my dissertation and the implications for the required software. It can be more or less stated that the computer offers a great diversity of possibilities to make interpreters' work easier, and this entails much more than simple terminology management. Several aspects developed in this dissertation have been confirmed in publications by experienced conference interpreters about the use of the computer in conference interpretation. The fact that requirements, especially in terminology management, differ considerably for translators and interpreters is also stated by Martin Will. He sees this difference mainly in the high level of concentration and thus limited attention capacity on the part of interpreters, especially during simultaneous interpretation in the booth. Multitasking is a crucial skill here. The visualisation of information on the computer screen and the structure of terminology entries need to be laid out accordingly (Will 2000: 125). Ralf Pfleger also stresses the importance of terminology work before, during and after the interpretation job in order to complete the information that can be found in dictionaries and other reference works. As a first step during preparation, difficult or striking elements are noted down in the mother tongue (with definition, context and source). These are then completed with the corresponding terms gathered from reading texts in the foreign language. Third, remaining gaps are filled through additional research (Pfleger 2001: 22). Interpreters have to provide for some extra time following conferences and work sessions for the integration of new terms noted during the conference as well as the overall maintenance of the terminology database.

A database for interpreters does not necessarily have to fulfil scientific requirements. It only needs to contain relevant information for their practical work. Information that can be found in existing reference works does not need to be integrated. Only the user himself or herself needs to be able and will be able to say in which context a special term occurred (Pfleger 2001: 23). 
Based on the thesis that a well-prepared interpreter is going to benefit from terminology resources system during simultaneous interpretation, requirements were developed for the terminology systems DolTerm and later LookUp (based on MS Access). It was observed that, when using this system during simultaneous interpretation, entering a term for query helped the interpreter to remember this term, and furthermore a quasi-active vocabulary was provided. Onomasiological structures may be useful for preparation purposes, but proved to be too slow for consultation in the booth and produced too high a cognitive burden. Research in the booth is limited to lexical gaps and is done according to semasiological or phonetic criteria. It could also be observed that associative clusters and terminology are organised in a chronological order according to the progress of the conference, probably in order to sort the terminology according to the logic of a presentation and in order to pre-activate the vocabulary (Stoll 2002: 35).

While at first glance it might be obvious to assume that, in terms of knowledge management, interpreters and translators have many things in common - after all, they both convey a message from one language to the other - the above findings reveal that in fact there are considerable differences.

When comparing the knowledge management needs of an interpreter to those of a translator, it can be stated that the translator's work focuses more on the translated text as such, whereas the interpreter has to rely more on efficient preparation and follow-up work in order to assure a certain quality in his or her work. Quick research and retrieval of documents and information is the first step, followed by filtering the relevant information and terminology, which can then be used for the preparation work such as memorising and organising knowledge. As there is not much time for recording valuable new knowledge gathered during a conference, follow-up plays a bigger role for interpreters than for translators, who can easily enter new terms in their personal databases while translating a text. Thus, the impact of an interpreter's terminological inventory stemming from the preparation and follow-up phase is much higher than that of a translator. Furthermore, due to the high concentration burden, the possibilities of looking up a term during interpretation are much more limited than for a translator. Access to information must be easier and more intuitive than for translators. Since conference interpreters, as opposed to translators, often work with more than one language pair, they also need structures that permit the visualisation of information in sometimes up to five, six or seven languages.

When comparing the knowledge management needs of an interpreter to those of a terminologist, it can be stated that the work of both consists in capturing data on unknown technical subjects, including relations between concepts. But an interpreter, as opposed to the terminologist, does not need complete or correct documentation or norms: even incorrect terms must be recorded if they are used by the speakers at a conference. The documentation of terminology is mainly a means for memorisation and consultation and 
ideally supports the interpreter in this task. This difference in purpose is also reinforced by the time pressure under which interpreters work.

\section{State of the art: software}

As to terminology management tools, some professional interpreters have developed their own terminology databases and do not use the existing commercial systems like MultiTerm or Term Star, which have been designed for translators' purposes. This fact alone shows that the standard terminology tools do not precisely serve interpreters' purposes. LookUp by Austermühl, Meyer und Stoll GbR (http://www.dolterm.de) offers the possibility of entering a term with its corresponding project, subject area, synonym/antonym, hyponym/hyperonym, definition, context, comment etc. and provides filters and queries for these information categories as well as a graphic representation of relations between terms. TermDB, a non-commercial interpreters' terminological database (MS Access-based) designed by Christian Vogeler, also offers data categories such as source, subject area and sub-area, customer, conference etc. plus filter and query functions. Interplex, the glossary software for interpreters and translators by Eric Hartner and Peter Sand (http://www.fourwillows.com/interplex/), has wide categorisation possibilities as well as a multi glossary import, search and visualisation function.

The above description of existing database solutions for use during simultaneous interpretation does not claim to be exhaustive or complete. But it shows that there is a need for special database systems for interpreters and that existing terminology management systems designed for translators do not seem to suit interpreters' purposes. The interpreters' databases developed so far all aim at facilitating use in the booth (especially via query and filter functions) and importing existing private glossaries. Structures are somewhat simpler than in "normal" terminology management systems like Trados or Term Star, and the visualisation of glossaries and search results is clearer. However, unlike these terminology management systems, which, in combination with a translation memory system, embrace the translator's whole workflow, i.e. from the first translation to the last spell and terminology check, and where terminology from the current translation can be easily entered in the terminology database and vice versa, the interpreters' databases do not include all relevant elements of an interpreter's workflow. For example, document management and especially the visualisation of parallel documents in several languages are not included, nor is the direct entry of terms from a text into the database. Terminology extraction, which is part of some terminology management systems, might also be a valuable function for conference interpreters.

Translators' terminology management systems offer flexible, vast and detailed entry possibilities (http://www.trados.com/library/documents/ MultiTerm/eng/ProdSumm_MT_TermWorkst_e.pdf ; http://www.star-solutions.net/html/dt/produkte/TransitXV-TerminologyMM.html). But this com- 
plexity sometimes makes them more complicated and slow to handle when it comes to generating word lists, filtering and sorting or representing entries or even relations between entries. Furthermore, the possibility of simultaneous research in several sources of information such as the internet, documents, dictionaries etc. might be a good feature that is still missing in both translators' and interpreters' terminology management systems. Especially for interpreters, when preparing for a conference and memorising the relevant terminology for it at short notice, training support functions could be helpful and cannot be found in either of the above-described database systems.

\section{A software model}

On the basis of the above-mentioned characteristics of and requirements for information research, organisation and acquisition in conference interpretation, deduced from relevant research findings and features of existing software, the structure of a possible software package covering the whole workflow can be derived. The following illustration and synoptic description of functions show a software model deduced from these requirements. This model consists of five modules and one central starting point.

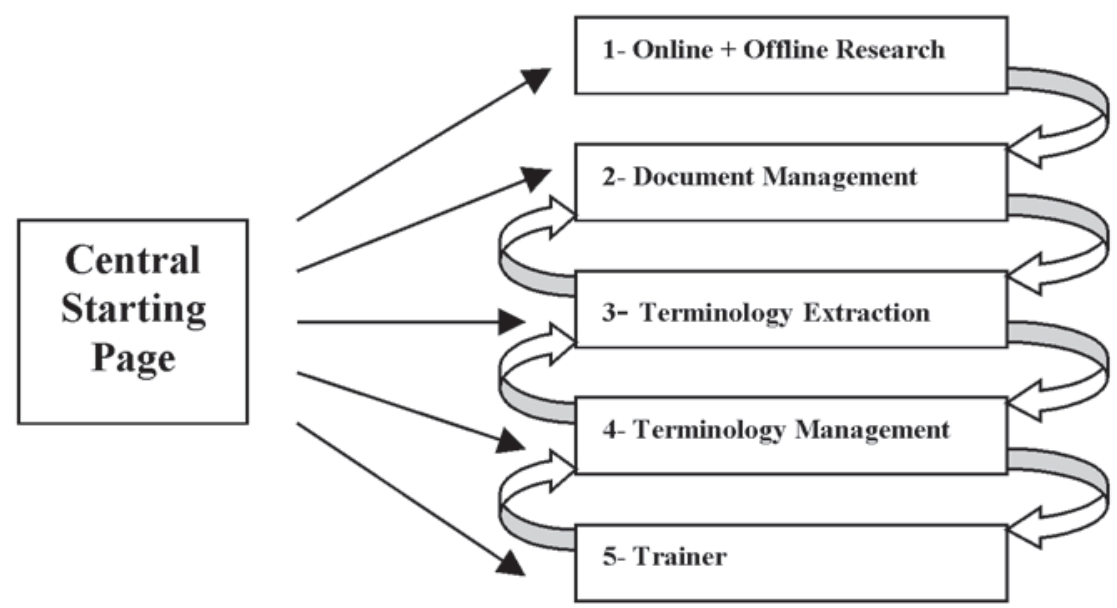

Module 1 - Online and Offline Research - finds internet documents of any kind about a particular subject (text, pictures, as well as glossaries and dictionaries) as well as documents already filed in Module 2 using keywords. It sorts the results by relevance and language.

Module 2 - Document Management - manages documents found in module 1, archives the interpreter's own documents, the ones received from the customer etc. This can be text, glossaries, graphs, bibliographies, summaries etc. Those documents can be found or sorted by subject, relevance to 
a certain subject, text type, language, date etc. Furthermore, status information from module 3 is saved in module 2, i.e. module 2 "remembers" from which document terminology has already been extracted (automatically or manually). It is also possible to indicate whether a text has already been read, where it comes from and when it was last used.

Furthermore, a connection between documents and term extraction and management could be useful in the form of information from modules 3 and 4 popping up when moving the mouse over a word or phrase. Parallel reading of texts in different languages can also be facilitated.

Module 3 - Terminology Extraction and Analysis - analyses documents (module 2), extracts potential technical terms and their equivalents in a different language when parallel texts in the respective languages are available or it is possible to consult electronic dictionaries or encyclopaedias. The result (term list) is then synchronised with module 4 - terminology management - in order to check which terms are already filed there. If a term is already registered in module 4 and it is marked as "known to the user", it is not put on the new list of extracted terms from module 3 . If it is filed in module 4, but not marked as "known to the user", it will appear on the list of extracted terms in module 3 , but there will be an indication saying that it is already registered in module 4 . Module 3 can also create word field lists for key subjects of a given text.

Module 4 - Terminology Management - manages entries (single words or whole sentences) coming from module 3 , those entered manually by the user and also entries imported from other databases (from colleagues, customers etc.). Such "external" data will of course be marked accordingly, making sure that it will not be integrated into the terminology database without being checked. Similar or double entries will also be marked and the user will be informed about them the moment they are entered into the database. Additional information can be entered if need be, like subject area (with no limit on the number of structure levels), project (conferences), source, grammatical category, degree of difficulty or importance, style, definitions, descriptions, context and graphs, also abbreviations and acronyms, date of entry, last access. The latter could be registered automatically. Relations can be established between different entries like "1 to 1 translation", "suggested translation", possible interference/faux amis, synonyms, antonyms, hyperonyms and hyponyms. Furthermore, status information from the training module (5) will be saved automatically. This information includes the date when an entry was last tested and whether it is actively or passively known or not known at all. Queries can be made individually ("looking up" morphological variants will also be recognised) and in the form of lists sorted and structured according to special criteria (conference, subject, degree of difficulty etc.) It is also possible to have a hierarchical representation of terminology of a certain subject area with the different subject levels.

Module 5 - the Trainer - helps systematic memorisation of terminology saved in module 4. A difference is made between entries that must be "drummed in", i.e. 1 to 1 equivalents between two languages, and transla- 
tions that are merely suggestions or elements that are translated differently in different situations or contexts. The latter are not "tested" in the sense that the user must render the exact equivalent but they are only "presented" visually or acoustically. The user's learning information is saved: unknown or problematic entries ("tip-of-the-tongue", tongue twisters or simply unusual or complicated expressions) are marked as such (manually by the user or automatically if the user fails the test). They will then be presented or tested automatically at regular intervals. This does not happen to known entries: they will remain untouched by the trainer except after a certain (userdefined) period of time. This function is particularly useful for the maintenance of a certain level of general language, a task often neglected in the heat of the moment of concrete conference preparation. The testing or presenting can be done in an ordered manner (subjects, degree of difficulty, projects etc.) or in a deliberately unsorted way. Finally, immediately before a conference, it is possible to print out a "last minute" list of very important or complicated or still unknown entries of a certain subject. Another useful function, especially for students, could be exercises aimed at practising interpretation-specific skills, e.g. clozing (visually or acoustically presented) or the presentation of word series that must be memorised and then rendered in a structured way.

In order to make it easier to search for a specific item during simultaneous interpretation in the booth, there should be an overall quick-search key that can be used blindly and independently of the module or function the user is using at that particular moment. When the user strikes this key and types the word or expression he or she is looking for (or part of it), all the modules are searched and the result in the respective (pre-defined) languages pops up in a big window showing all the results in a clearly legible and well-structured way. Different colours for different languages (user-defined) might be helpful to grasp the search result at one glance. With another key strike, the window will close again. So if, for example, the interpreter was reading a document which is being discussed in the conference in module 2, he or she can intuitively strike the search key, find the respective word and translation and then strike the close-search key in order to go on reading the text.

\section{Conclusion and perspectives}

It has clearly been shown that conference interpreters do need specialised software solutions, as their needs differ from those of translators or terminologists, and therefore existing software designed for the latter does not suit their purposes. The fact that databases for terminology management in conference interpreting have already been designed underpins this conclusion.

Having taken a closer look at scientific findings in this field and the differences between the needs of interpreters and those of related professions like translators or terminologists, it becomes clear in what sense conference interpreters need special software. The software model described in section 
4 translates the interpreter's requirements identified in sections 2 and 3 into a representation of an electronic information manager.

This software model is based on findings, which, however, do not always come from interpretation research as such but from neighbouring disciplines. This picture still needs to be completed and refined.

Apart from the more technical aspects, there are some key areas of interest that deserve further investigation. For example, how should relations between terminological entries be organised? There are various kinds of relevant relations in the terminological inventory of an interpreter: conceptual relations like hyperonyms/hyponyms and meronyms/holonyms, semantic relations between denominations like synonyms and antonyms, also more subjective relations like classification in different subject areas or projects, faux amis and many more. Here, the question of multiple classification is also relevant. A second item of interest is what the learning methods used by conference interpreters are when preparing for a technical conference? Are there general "patterns"? Could they be optimised? Do interpreters ever dedicate some of their time to long-term preparation and maintenance of information in their mother tongue and in foreign languages? Is self-discipline a problem here and if yes, how could it be tackled? A different issue still is where to draw the limit between individual databases and general (public) ones? Does an interpreter have to save every detail that comes up in the context of a conference in his personal database, or is it sometimes enough to know where to find it (dictionaries, internet, other people's glossaries)? Or might a kind of "bookmarking" be more useful? What kind of information is likely to be useful later on, what is not? For example, in a conference about agricultural statistics, a list of 20 different types of fruits and vegetables is discussed. The interpreter, eager to know all the 20 words in all his working languages (two, three, four or more), puts all the terms into his database. The following week, he works at a conference about fishery - 20 different fish names occur. Will all this very special terminology (have to) be memorised? Does it all have to figure in the interpreter's database if he can also find the terms in his electronic dictionaries? Does the structure of the database have any impact on these questions? Is it not naive to believe that we will remember a term just because we have it in our database? What does the optimum follow-up after a conference look like? What impact does it have on the quality of the interpretation in another conference on the same subject? What impact does it have on the quality of the interpretation in another conference on a different subject?

IT offers promising possibilities, so there is a chance that we might someday be able to make our computer act like an infallible, perfectly organised, nearly all-knowing, but still discreet butler who reminds us not to forget our keys (keywords) when leaving the house, who finds the shoes that match our dress (the word we are looking for) in the remotest corner of our wardrobe, who keeps our place tidy and teaches us where to put our stuff in order to be able to find it when we need it. This could help us to concentrate more on the task of interpretation as such, also in the training phase, whilst 
being more efficient, less stressed and obtaining an even better grasp of the (technical) language and underlying knowledge.

After all, interpreters and butlers have one thing in common: They are at their best when you do not even notice they are around. So they should be a perfect team.

\section{Bibliography}

Austermühl, Meyer und Stoll GbR. „LookUp.“ Online at http://www.dolterm.de (consulted 13 August 2004)

Hartner, E. and P. Sand "Interplex - Glossary Software for Interpreters and Translators." On line at http://www.fourwillows.com/interplex/ (consulted 13 August 2004)

Pfleger, R. (2001). "Was man nicht im Kopf hat, muss man in der Datenbank haben! Terminologiearbeit aus der Sicht eines Dolmetschers." MDÜ 1/2001, $22-23$

Rütten, A. (2000). "Informationsmanagement für Dolmetscher - Anforderungen an spezielle Software zur Konferenzvorbereitung" Saarbrücker Studien zu Sprachdatenverarbeitung und Übersetzen, Volume 17

Star AG. "Transit XV. Terminologiemanagement." Online at http://www.star-solutions.net/html/dt/produkte/TransitXV-TerminologyMM.html (consulted 13 August 2004)

Stoll, C. (2002). “Terminologiesysteme für Simultandolmetscher.” In: Mayer, F. and K.-D. Schmitz and J. Zeumer (ed.). eTerminology. Akten des Symposions, Köln, 12-13 April 2002. Cologne: Deutscher Terminologie-Tag e.V., $31-42$

Trados Incorporated. "Product Summary Multiterm." Online at http://www.trados.com/library/documents/MultiTerm/eng/ProdSumm_MT_TermWorkst_e. pdf (consulted 13 August 2004)

Will, M. (2000). "Bemerkungen zum Computereinsatz beim Simultandolmetschen." Kalina, S. and S. Buhl and H. Gerzymisch-Arbogast (Hgg.). Dolmetschen: Theorie • Praxis • Didaktik - mit ausgewählten Beiträgen der Saarbrücker Symposien. Arbeitsberichte des Advanced Translation Research Center (ATRC) an der Universität des Saarlandes. St. Ingbert: Röhrig Universitätsverlag, 125-135. 
